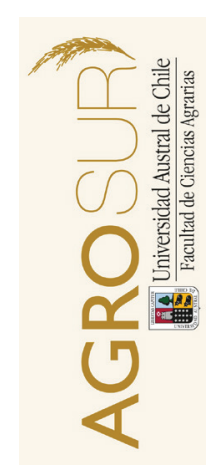

\section{¿Cambia la mineralización del nitrógeno desde abonos orgánicos compost, bokashi y biol provenientes del mismo guano de gallina?}

\author{
Does nitrogen mineralization change in organic fertilizers compost, \\ bokashi and biol from the same poultry guano?
}

\author{
Cárdenas, C. ${ }^{a}$, Carrasco, J. ${ }^{a}$, Pinochet, D. ${ }^{a, b}$
}

${ }^{a}$ Instituto de Ingeniería Agraria y Suelos, Universidad Austral de Chile. Casilla 567, Valdivia, Chile.

${ }^{b}$ Centro de Investigación en Suelos Volcánicos, Universidad Austral de Chile. Casilla 567, Valdivia, Chile.

\begin{tabular}{l} 
A R T I C L E I N F O \\
\hline Keywords: \\
Organic fertilizers \\
Macronutrients \\
Mineralization \\
\hline
\end{tabular}

Original Research Article, Soil Science

*Corresponding author:

Dante Pinochet

E-mail address:

dpinoche@uach.cl

\begin{abstract}
A B S T R A C T
The origin of the materials that make up the organic fertilizers and the process of their elaboration, determine the final nutritional characteristics of the product. The objective of this study was to evaluate the nutritional composition of three organic fertilizers from the same source of material. Compost, bokashi and biol, were produce under controlled conditions of humidity and temperature from poultry guano as source material. The organic fertilizers were incubated at $28 \pm 1{ }^{\circ} \mathrm{C}$ for 18 weeks, to quantify the potentially mineralizable nitrogen $(\mathrm{N})$. The initial nutritional characterization of fertilizers showed that poultry and biol presented the highest percentages of macronutrients ( $p>0.05)$. The highest mineralization rate was found in poultry $\left(5.19 \%\right.$ organic $N$ week $\left.{ }^{-1}\right)$, similar to compost, $5.17 \%$ organic $\mathrm{N}$ week ${ }^{-1}$, and both higher than bokashi $\left(4.47 \% \mathrm{~N}\right.$ organic week $\left.{ }^{-1}\right)$. The biol was adjusted to a sigmoidal equation, with a mineralization rate of $1.6 \%$ organic $\mathrm{N}_{\text {week }}{ }^{-1}$, after $50 \%$ of the original $\mathrm{N}$ content has been mineralized. The accumulated mineralized $\mathrm{N}$ varied from 11620 $\mathrm{mg} \mathrm{kg}^{-1}$ to $2138 \mathrm{mg} \mathrm{kg}^{-1}$ in the four fertilizers, showing that the main component of accumulated mineralized $\mathrm{N}$ is in the form of ammonium and of the four fertilizers, biol was the one that presents the highest proportion of mineralized $\mathrm{N}$ as nitrate.
\end{abstract}

\title{
RESUMEN
}

El origen delos materiales que componen los fertilizantes orgánicos y el proceso de su elaboración, determinan las características finales del producto. El objetivo de este estudio fue evaluar la composición nutricional de tres fertilizantes orgánicos provenientes del mismo material de origen: compost, bokashi y biol. Estos fueron elaborados en condiciones controladas de humedad y temperatura a partir de guano de gallina, como material de origen. Los fertilizantes orgánicos fueron incubados a $28 \pm 1{ }^{\circ} \mathrm{C}$ por 18 semanas, para cuantificar el nitrógeno $(\mathrm{N})$ potencialmente mineralizable. La caracterización nutricional inicial de los abonos mostró que, el guano de gallina y el biol, presentaron los mayores porcentajes de macronutrientes ( $p>0,05)$. La mayor tasa de mineralización se presentó en el guano de gallina (5,19\% $\mathrm{N}$ orgánico semana-1), similar a la del compost, $5,17 \% \mathrm{~N}$ orgánico semana-1 ${ }^{-1}$ y ambos mayores a la del bokashi $\left(4,47 \% \mathrm{~N}\right.$ orgánico semana $\left.{ }^{-1}\right)$. La mineralización del biol fue ajustada a una ecuación sigmoidea, mostrando una tasa de mineralización de 1,6\% $\mathrm{N}$ orgánico semana-1 ${ }^{-1}$ después de que se ha mineralizado el 50\% del contenido de $\mathrm{N}$ original. El $\mathrm{N}$ mineralizado acumulado varió desde 11,6 g kg-1 a 2,1 g kg-1 en los cuatro fertilizantes, mostrando que el componente principal del $\mathrm{N}$ mineralizado acumulado está en la forma de amonio y de los cuatro fertilizantes biol, es quien presenta la mayor proporción del N mineralizado como nitrato.

Palabras clave: fertilizantes orgánicos, macronutrientes, mineralización.

\section{INTRODUCCIÓN}

En la agricultura orgánica se emplean diversos fertilizantes y abonos cuya composición varía dependiendo del origen de producción y la fuente de nutrientes (Altieri y Nicholls, 2012), además, del grado de procesamiento y el estado físico del fertilizante (Ramos y Terry, 2014). El guano de gallina es uno de los abonos sólido sin procesar más comunes (Yagodín et al., 1986), caracterizado por presentar concentra- ciones de nutrientes relativamente mayores que otros fertilizantes orgánicos, aportando nitrógeno, fósforo, potasio, calcio, magnesio, hierro, principalmente (Restrepo, 2007). También el guano de gallina es utilizado como base para elaborar otros abonos orgánicos procesados sólidos como compost, bokashi o líquidos como biol (Varnero et al., 2002). Estos abonos elaborados usualmente tienen una mejor apariencia física que los materiales originales que lo componen, utilizándose descomposiciones aeróbicas como anaeróbicas en 
la mineralización de los materiales originales (Guanopatín, 2012).

El nitrógeno $(\mathrm{N})$ es uno de los elementos de mayor interés para la fertilización, debido a que es un macronutriente primario, que indica que es de los primeros nutrientes en ser deficiente en los agroecosistemas, pero que un exceso puede causar fácilmente contaminación ambiental (Jones et al., 2017). En los fertilizantes orgánicos, el $\mathrm{N}$ está en forma orgánica, debiendo ser transformado a formas inorgánicas, para que la planta pueda asimilarlo; este proceso se conoce como mineralización (Havlin et al., 1990). La tasa de mineralización está determinada por la humedad, temperatura, aireación, razón $\mathrm{C} / \mathrm{N}$ y pH; por lo cual, su uso eficiente dependerá de las condiciones de la zona edafoclimáticas donde se aplique el fertilizante y del momento de la aplicación (Kameko et al., 2003). Por ello, la aplicación de fertilizantes orgánicos en la agricultura está limitada cuando se desconoce la tasa de mineralización que presentan estos abonos, lo que usualmente lleva a subestimar o sobrestimar la dosis a aplicar. Una subestimación afecta el rendimiento del cultivo y con ello la rentabilidad en el sistema productivo, y un exceso, provocará pérdidas contaminantes a través de la lixiviación de $\mathrm{NO}_{3}$ a aguas profundas y la desnitrificación a $\mathrm{N}_{2} \mathrm{O}$ que es un gas de efecto invernadero (Clivot et al., 2017). Por ello, para la aplicación adecuada de estos abonos se requiere conocer la curva de mineralización de N (Figueroa-Barrera et al., 2012). Así, el objetivo de este trabajo es caracterizar tres abonos orgánicos elaborados con la misma materia prima básica, guano de gallina y evaluar comparativamente la mineralización potencial de $\mathrm{N}$ de los abonos orgánicos producidos.

\section{MATERIAL Y MÉTODOS}

\section{Composición de los abonos orgánicos}

Los abonos orgánicos fueron preparados considerando la producción de $10 \mathrm{~kg}$, en base seca, para compost y bokashi y de 10 litros de biofermento para biol. La fuente principal de $\mathrm{N}$ en los tres abonos orgánicos evaluados fue guano de gallina, tamizado a $4 \mathrm{~mm}$. Se extrajeron los restos de plumas y otros elementos externos en forma manual, con la finalidad de dar homogeneidad al guano. La participación del guano de gallina en los abonos elaborados, en base seca, fue: $40 \%$ en compost (Barbaro et al., 2013), 33,3\% en bokashi (Infante, 2011) y 25\% para biol (Suquilandia, 1996; Guanopatín, 2012). El guano de gallina (100\%) constituyó el tratamiento control. Los demás materiales usados y su aporte para la elaboración como abono orgánico son resumidos en el Cuadro 1. La elaboración de los fertilizantes orgánicos se realizó en un recinto cerrado en la localidad de Punucapa, Valdivia.
Cuadro 1. Resumen de los materiales utilizados en la elaboración de los abonos orgánicos.

Table 1. Summary of the materials used in the elaboration of organic fertilizers.

\begin{tabular}{lccc}
\hline & \multicolumn{3}{c}{ Abonos orgánicos } \\
\cline { 2 - 4 } & Compost & Bokashi & Biol \\
\hline Afrechillo (\%) & 30 & 33 & N.O \\
Guano (\%) & 40 & 33 & 25 \\
Suelo (\%) & 10 & 33 & N.O \\
Paja (\%) & 20 & N.O & N.O \\
Levadura (gramos) & N.O & 100 & 100 \\
Leche (litros)* & N.O & 3 & 3 \\
Miel (gramos)* & N.O & 125 & 125 \\
\hline
\end{tabular}

* En el caso de la levadura, la leche y la miel adicionadas, el porcentaje de composición fue en base a los materiales afrechillo, guano, suelo y paja para constituir el $100 \%$. El porcentaje de utilización de cada material fue consultado en la literatura disponible (Varnero et al., 2002; Zbytniewski y Buszewski, 2005; Infante, 2011; Guanopatín, 2012; SAG, 2013). N.O: no se ocupó.

\section{Elaboración de los abonos orgánicos derivados de guano de gallina}

Compost. Fue elaborado en recipientes de 20 litros, perforados en la parte lateral e inferior, para asegurar la oxigenación y el escurrimiento de líquidos. Los materiales originales se mezclaron y homogeneizaron (afrechillo, guano de gallina, suelo y paja) e hidratados a un $60 \%$. El proceso de compostaje se dio por finalizado a los 54 días, cuando se cumplió con el criterio de NCh2880 (SAG, 2013) que exige para finalización del proceso una temperatura de compostaje mínima de $55^{\circ} \mathrm{C}$ por 3 días consecutivos o de $45^{\circ} \mathrm{C}$, por 12 días consecutivos.

Bokashi. Fue elaborado envuelto en un plástico negro (para proteger de la luz y condiciones externas) homogenizando afrechillo, guano de gallina y suelo; a estos materiales, se les añadió una mezcla, realizada previamente, de leche, miel y levadura ("solución fermentadora"). Se hidrató hasta lograr un $60 \%$ de humedad y se dejó fermentar por los 13 días, hasta que alcanzó un color grisáceo y un bajo contenido de humedad.

Biol. Se utilizó recipientes fermentadores de 5 litros. Se introdujeron 1,7 kilos de guano de gallina, 1 litro de la solución fermentadora y agua hasta completar el volumen. Los recipientes tenían una vía de salida de gases, conectada a un filtro de agua, que actuó como indicador al término del proceso de fermentación. Ello ocurrió a los 37 días, indicado por la ausencia de burbujeo en el filtro de agua (Infante, 2011). 
Finalizada la elaboración de los tres abonos orgánicos, elaborados con guano de gallina, se homogeneizaron y en cada uno de ellos se tomaron 3 submuestras de aproximadamente $2 \mathrm{~kg}$, para realizar las caracterizaciones y el estudio de mineralización.

Para la caracterización de los abonos se analizó el contenido de materia orgánica (MO) por calcinación y pérdida de peso, carbono orgánico (digestión húmeda), nitrógeno total por Kjeldahl, y en cenizas el contenido total de los nutrientes minerales $\mathrm{P}, \mathrm{K}, \mathrm{Ca}, \mathrm{Mg}, \mathrm{Na}, \mathrm{Cu}, \mathrm{Fe}$, $\mathrm{Mn}, \mathrm{Zn}$ y Al mineral, métodos descritos en Sadzawka et al. (2005). La determinación de la relación C/N, conductividad eléctrica, $\mathrm{pH}$ y los contenidos totales de los diferentes nutrientes fueron expresados en base materia seca $\left(105^{\circ} \mathrm{C}\right)$, de acuerdo de los protocolos de análisis de compost descritos por Sadzawka et al. (2005).

El nitrógeno potencialmente mineralizable (Npm) de los abonos orgánicos sólidos, fue determinado incubando muestras en tubos de lixiviación de $50 \mathrm{~mL}$, recubiertos con lana de vidrio en la parte inferior; a los cuales, se les introdujo una mezcla de $10 \mathrm{~g}$ de cada abono orgánico con $20 \mathrm{~g}$ de cuarzo tamizado a $2 \mathrm{~mm}$; luego, fueron hidratadas a un $60 \%$ de humedad base seca. El N mineral inicial de las muestras fue lavado con $100 \mathrm{~mL}$ de $\mathrm{CaCl}_{2}$ 0,01 M, seguido por la adición de $20 \mathrm{~mL}$ de solución nutritiva sin $\mathrm{N}$, compuesta por las siguientes sales: $\left(\mathrm{CaSO}_{4}\right)_{2} \cdot 2 \mathrm{H}_{2} \mathrm{O} 0,002 \mathrm{M} ; \mathrm{MgSO}_{4} 0,002 \mathrm{M} ; \mathrm{Ca}\left(\mathrm{HPO}_{4}\right)_{2} \cdot \mathrm{H}_{2} \mathrm{O}$ $0,005 \mathrm{M} \mathrm{y} \mathrm{K}_{2} \mathrm{SO}_{4} 0,0025 \mathrm{M}$. Este procedimiento se repitió después de cada lixiviación, dentro del periodo de incubación. Después de cada lavado, el exceso de agua de la muestra fue removido mediante vacío, hasta que las muestras dejaron de drenar. Los tubos fueron cubiertos con polietileno e incubados a $28^{\circ} \mathrm{C} \pm 1{ }^{\circ} \mathrm{C}$ por 18 sema- nas. La extracción mediante lixiviación del $\mathrm{N}$ mineralizado se realizó a las semanas 1, 2, 3, 4, 5, 6, 7, 8 y 18 de incubación. Finalmente, el $\mathrm{N}$ mineral extraído en los $100 \mathrm{~mL}$ $\mathrm{CaCl}_{2}$, fue determinado por destilación con arrastre de vapor (Stanford y Smith, 1972; Pinochet et al., 2000).

El Biol, fue puesto en la cámara de incubación en vasos de $50 \mathrm{~mL}$ cubiertos con polietileno en el mismo momento que los abonos sólidos. En cada muestreo se le extrajo $100 \mathrm{~mL}$ para la determinación por destilación con arrastre de vapor, adaptado del método descrito por Pinochet et al. (2000).

\section{Análisis estadístico}

La variación en la composición química inicial de los fertilizantes orgánicos y del guano de gallina utilizado como control, se evaluó con un análisis de varianza (ANDEVA) y separación de medias usando Tukey HSD, con un intervalo de confianza de un 95\%. Para evaluar el comportamiento de la mineralización de $\mathrm{N}$ en el tiempo, se acumularon los valores de $\mathrm{N}$ mineralizado semanalmente. Las curvas acumuladas fueron descritas por regresión no lineal, con el programa GraphPad Prism v.6.0, en función del tiempo de incubación.

\section{RESULTADOS}

\section{Caracterización química de los fertilizantes orgáni- cos analizados}

El mayor porcentaje de $\mathrm{N}$ fue mayor en biol y guano de gallina, que fueron iguales $(p>0,05)$. Se diferenciaron de compost $(2,75 \%)$ y bokashi $(1,88 \%)$ (Figura 1$)$.

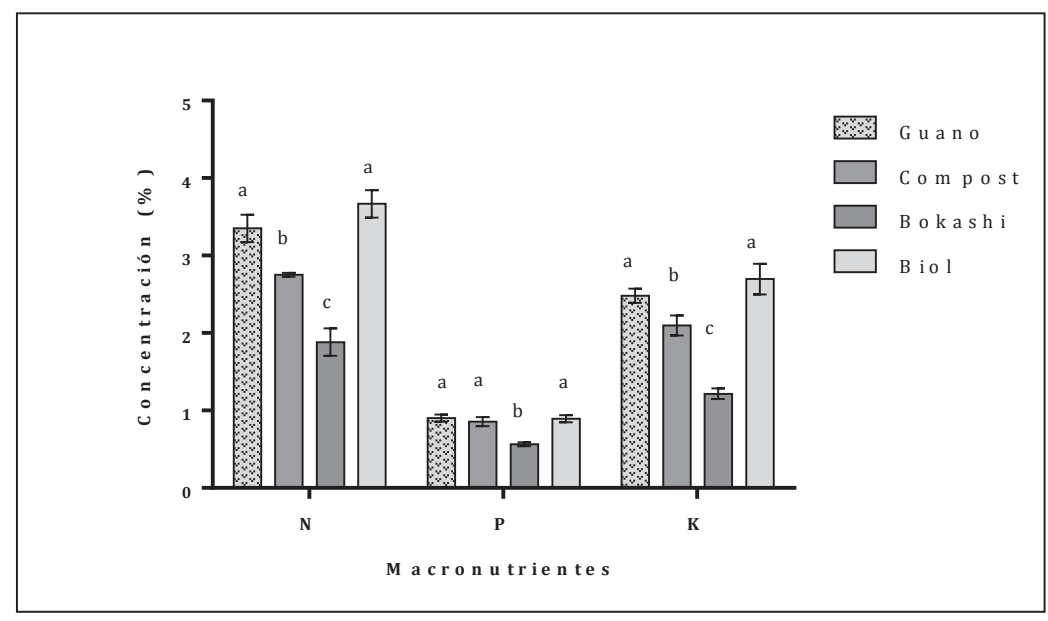

Figura 1. Contenido total de macronutrientes para los cuatro fertilizantes orgánicos evaluados. Las letras indican diferencias significativas entre los porcentajes de nutrientes presentes en los fertilizantes $(p<0,05)$. Barras verticales muestran error estándar de la media.

Figure 1. Total content of macronutrients for the four organic fertilizers evaluated. The letters indicate significant differences between the percentages of nutrients present in the fertilizers $(\mathrm{p}<0.05)$. Vertical bars show standard error of the mean. 
El contenido de fósforo no fue diferente entre guano de gallina, compost y biol ( $p>0,05)$; bokashi fue quien presentó el menor valor $(0,56 \%$ de $\mathrm{P})$, de todos los fertilizantes evaluados (Figura 1). Potasio (K) se presentó en mayor porcentaje en guano y biol, sin diferencias significativas entre ellos ( $p>0,05)$, seguido por compost $\mathrm{y}$, en un nivel inferior de bokashi $(1,20 \%$ de K) a los otros tres abonos orgánicos (Figura 1).

Con respecto a los cationes presentes en los abonos evaluados (Cuadro 2), bokashi es quien presenta contenidos superiores de $\mathrm{Al}$ y micronutrientes cationes $(\mathrm{Fe}, \mathrm{Mn}$, $\mathrm{Cu}$ y $\mathrm{Zn}$ ) diferenciándose, en general, del compost quien, a su vez, presentó los contenidos mayores de Ca y Mg. El contenido el biol $(p<0,05)$, en general, fue el que presentó menores niveles de todos los cationes evaluados (Cuadro 2). El catión Na fue el único que no se diferenció entre todos los fertilizantes orgánicos, presentando un contenido relativamente bajo $(0,1 \%$ de $\mathrm{Na})$. La presencia de micronutrientes fue en concentraciones bajo la norma Chilena 2880.

La preparación de los fertilizantes orgánicos afectó su contenido de MO $(\mathrm{p}<0,05)$. El mayor contenido de MO se presentó en biol (78\%), quien fue superior en 1,16 veces a guano, en 1,42 veces a compost y 1,77 veces a bokashi (Figura 2).

Los cuatro fertilizantes orgánicos, fueron distintos $(\mathrm{p}<0,05)$ en el $\mathrm{pH}$, variando en rango cercano a la neutralidad $(7,4)$ tres de ellos y siendo solo biol ligeramente ácido $(5,3)$ (Cuadro 3). Por el contrario, el mayor valor de CE fue en biol $\left(21,1 \mathrm{dS} \mathrm{m}^{-1}\right)$, lo cual es muy alto. Los demás fertilizantes orgánicos presentaron valores de CE de 3 a 4 veces menores que biol (Cuadro 3). La relación $\mathrm{C} / \mathrm{N}$ varió entre los fertilizantes orgánicos (11 y 13 aproximadamente) pero su rango cualitativo fue similar (Cuadro 3).
El contenido de $\mathrm{N}$ mineral inicial (Nmi) fue incluido como una medida de la mineralización del $\mathrm{N}$ orgánico, durante el proceso de preparación del fertilizante. Biol presentó la mayor proporción de $\mathrm{N}$ mineralizado en su preparación, siendo 1,5 veces superior a guano de gallina y por sobre 6,0 veces a compost y bokashi (Cuadro 3), ya que compost y bokashi no mostraron diferencias entre sí $(p>0,05)$.

\section{Nitrógeno potencialmente mineralizable (Npm)}

Se observaron diferentes tasas de mineralización de Npm, después del ajuste de la misma ecuación exponencial de mineralización para el guano de gallina, compost y bokashi. Guano y compost presentaron similares tasas constantes de mineralización por semana, alrededor del 5,2\%, mientras que el bokashi fue ligeramente menor (4,5\%). El biol, por su parte, fue ajustado a una ecuación logística tipo Boltzman, mostrando una tasa constante de mineralización de $1,6 \%$ por semana después de que se ha mineralizado el $50 \%$ del contenido de $\mathrm{N}$ original (Figura 3). Los fertilizantes orgánicos evaluados presentaron diferencias en la cantidad total de $\mathrm{Npm}$, variando 5,5 veces entre ellos, desde 11,6 g $\mathrm{kg}^{-1}$ de Npm para el guano de gallina a 2,1 $\mathrm{g} \mathrm{kg}^{-1}$ en biol. Los fertilizantes orgánicos sólidos estabilizaron la mineralización a la octava semana, en tanto biol presentó una fase lag de cerca de 4 semanas, a partir de la cual comienza una mineralización que alcanza una estabilidad cerca de las 12 semanas (Figura 3).

La alta correlación lineal entre el $\mathrm{N}$ mineralizado acumulado total y el $\mathrm{N}$ mineral amoniacal, indica que casi la totalidad del $\mathrm{N}$ mineralizado en bokashi (98\%), el $85 \%$ en guano y el $90 \%$ en compost se encontró en

Cuadro 2.Contenido total de nutrientes cationes y minerales $\left(\mathrm{mg} \mathrm{kg}^{-1}\right)$ presentes en los cuatro fertilizantes orgánicos evaluados.

Table 2. Total content of nutrients cations and minerals $\left(\mathrm{mg} \mathrm{kg}^{-1}\right)$ present in the four organic fertilizers evaluated.

\begin{tabular}{lcccc}
\hline Minerales & Guano & Compost & Bokashi & Biol \\
\cline { 2 - 5 } & \multicolumn{4}{c}{ (mg kg-1) } \\
\hline Aluminio & $4003,4 \pm 102,5 \mathrm{c}$ & $7034,5 \pm 391,1 \mathrm{~b}$ & $14251,6 \pm 347,0 \mathrm{a}$ & $3208,3 \pm 49,9 \mathrm{c}$ \\
Hierro & $227,2 \pm 4,5 \mathrm{c}$ & $9306,3 \pm 396,1 \mathrm{~b}$ & $16962,5 \pm 955,3 \mathrm{a}$ & $179,1 \pm 8,1 \mathrm{c}$ \\
Sodio & $1103,8 \pm 16,7 \mathrm{a}$ & $1183,8 \pm 7,6 \mathrm{a}$ & $1035,4 \pm 34,3 \mathrm{a}$ & $1083,3 \pm 72,9 \mathrm{a}$ \\
Calcio & $3166,7 \pm 120,1 \mathrm{~b}$ & $13000,0 \pm 172,1 \mathrm{a}$ & $3666,3 \pm 238,5 \mathrm{~b}$ & $2916,7 \pm 145,8 \mathrm{~b}$ \\
Magnesio & $4031,3 \pm 93,8 \mathrm{~b}$ & $4875,0 \pm 126,3 \mathrm{a}$ & $4531,3 \pm 82,7 \mathrm{a}$ & $2812,5 \pm 65,1 \mathrm{c}$ \\
Cobre & $25,2 \pm 0,8 \mathrm{c}$ & $31,1 \pm 1,1 \mathrm{~b}$ & $39,7 \pm 0,5 \mathrm{a}$ & $20,1 \pm 0,7 \mathrm{~d}$ \\
Manganeso & $136,9 \pm 2,3 \mathrm{~b}$ & $149,8 \pm 6,9 \mathrm{~b}$ & $438,5 \pm 16,3 \mathrm{a}$ & $119,3 \pm 3,8 \mathrm{~b}$ \\
Zinc & $6,5 \pm 0,3 \mathrm{~b}$ & $142,5 \pm 11,9 \mathrm{a}$ & $131,3 \pm 0,7 \mathrm{a}$ & $6,7 \pm 0,5 \mathrm{~b}$ \\
\hline
\end{tabular}

Las letras indican diferencias significativas entre los contenidos de nutrientes presentes en los fertilizantes $(\mathrm{p}<0,05)$. 


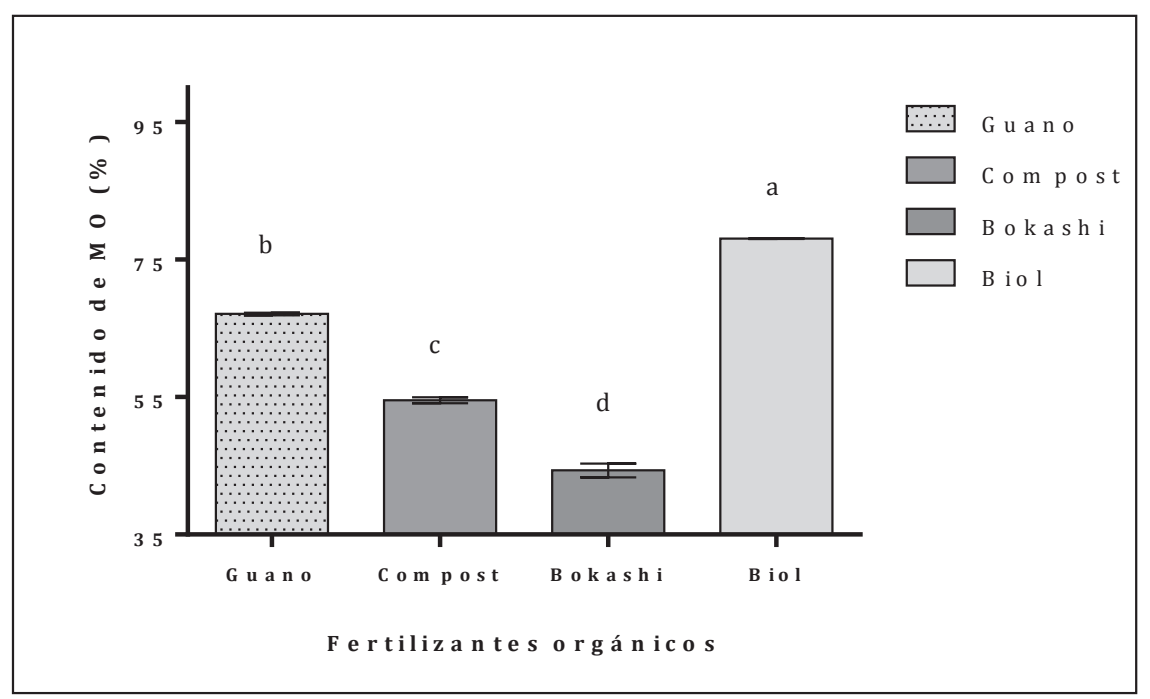

Figura 2. Contenido porcentual de materia orgánica (MO) para los cuatro fertilizantes orgánicos caracterizados, las letras indican diferencias significativas entre los contenidos de MO presentes en los fertilizantes $(\mathrm{p}<0,05)$. Barras verticales muestran error estándar de la media.

Figure 2. Percentage organic matter (OM) content for the four organic fertilizers characterized, the letters indicate significant differences between the contents of MO present in the fertilizers $(\mathrm{p}<0.05)$. Vertical bars show standard error of the mean.

Cuadro 3. Valores de pH, conductividad eléctrica, relación $\mathrm{C} / \mathrm{N}$ y N mineral inicial en los abonos orgánicos.

Table 3. Values of $\mathrm{pH}$, electrical conductivity, $\mathrm{C} / \mathrm{N}$ ratio and $\mathrm{N}$ mineral initial in organic fertilizers.

\begin{tabular}{|c|c|c|c|c|}
\hline & Guano & Compost & Bokashi & Biol \\
\hline pH dilución (1:5) & $6,82 \pm 0,03 \mathrm{~b}$ & $7,40 \pm 0,01 \mathrm{a}$ & $6,62 \pm 0,01 \mathrm{c}$ & $5,32 \pm 0,01 d$ \\
\hline $\mathrm{CE}\left(\mathrm{dS} \mathrm{\textrm {m } ^ { - 1 } )}\right.$ & $5,57 \pm 0,04 c$ & $7,33 \pm 0,03 b$ & $5,44 \pm 0,012 \mathrm{c}$ & $21,14 \pm 0,54 \mathrm{a}$ \\
\hline Relación C/N & $11,04 \pm 0,13 a$ & $11,14 \pm 0,34 \mathrm{a}$ & $13,04 \pm 0,42 a$ & $11,80 \pm 0,32 a b$ \\
\hline $\mathrm{N}$ inicial (mg kg-1) & $1396 \pm 83 b$ & $341 \pm 11 \mathrm{c}$ & $238 \pm 6 c$ & $2125 \pm 27 a$ \\
\hline
\end{tabular}

Las letras indican diferencias significativas entre los fertilizantes $(\mathrm{p}<0,05)$, para cada variable. CE: conductividad eléctrica.

forma de amonio. Por su parte, biol presentó cerca del $60 \%$ como amonio y un $40 \%$ de nitratos (Figura 4 ).

\section{DISCUSIÓN}

\section{Composición química de los fertilizantes orgánicos}

Varios trabajos han mostrado que la proporción de materias primas utilizadas en la elaboración de los fertilizantes orgánicos, influyen fuertemente en la calidad nutricional (Leblanc et al., 2007; Pérez et al., 2008) y en la participación final de los macronutrientes primarios en los fertilizantes orgánicos (Restrepo, 2009). Similarmente, en este estudio, el contenido de $\mathrm{N}$ en los abonos fue proporcional a la cantidad de guano de gallina utilizado en su preparación $(100 \%$, en guano y biol, $40 \%$ compost y $33 \%$ en bokashi). La disminución del contenido de guano fue en función del aumento de otros componentes como materiales vegetales. Se ha mostrado que los abonos orgánicos como compost y bokashi preparados con un mayor contenido de materiales vegetales $(>30 \%)$, presentan un menor aporte de los nutrientes N, P, K (Castillo et al., 2000), lo que fue coincidente con los resultados de este estudio.

Se ha mostrado que la elaboración de un abono orgánico incrementa la mineralización, cambiando la concentración original de nutrientes desde la fuente (Díaz et al., 2004), lo que es usual en la preparación de compost, tal como se observó en este estudio. Así, los minerales cationes como $\mathrm{Ca}, \mathrm{Mg}$, $\mathrm{Fe}, \mathrm{Mn}$ y $\mathrm{Al}$ se concentraron más en compost y bokashi, coincidiendo con el aumento de otras materias primas distintas al guano de gallina (paja, suelo y afrechillo). La variabilidad del Ca y $\mathrm{Mg}$ observada ha sido explicada por la presencia de mate- 


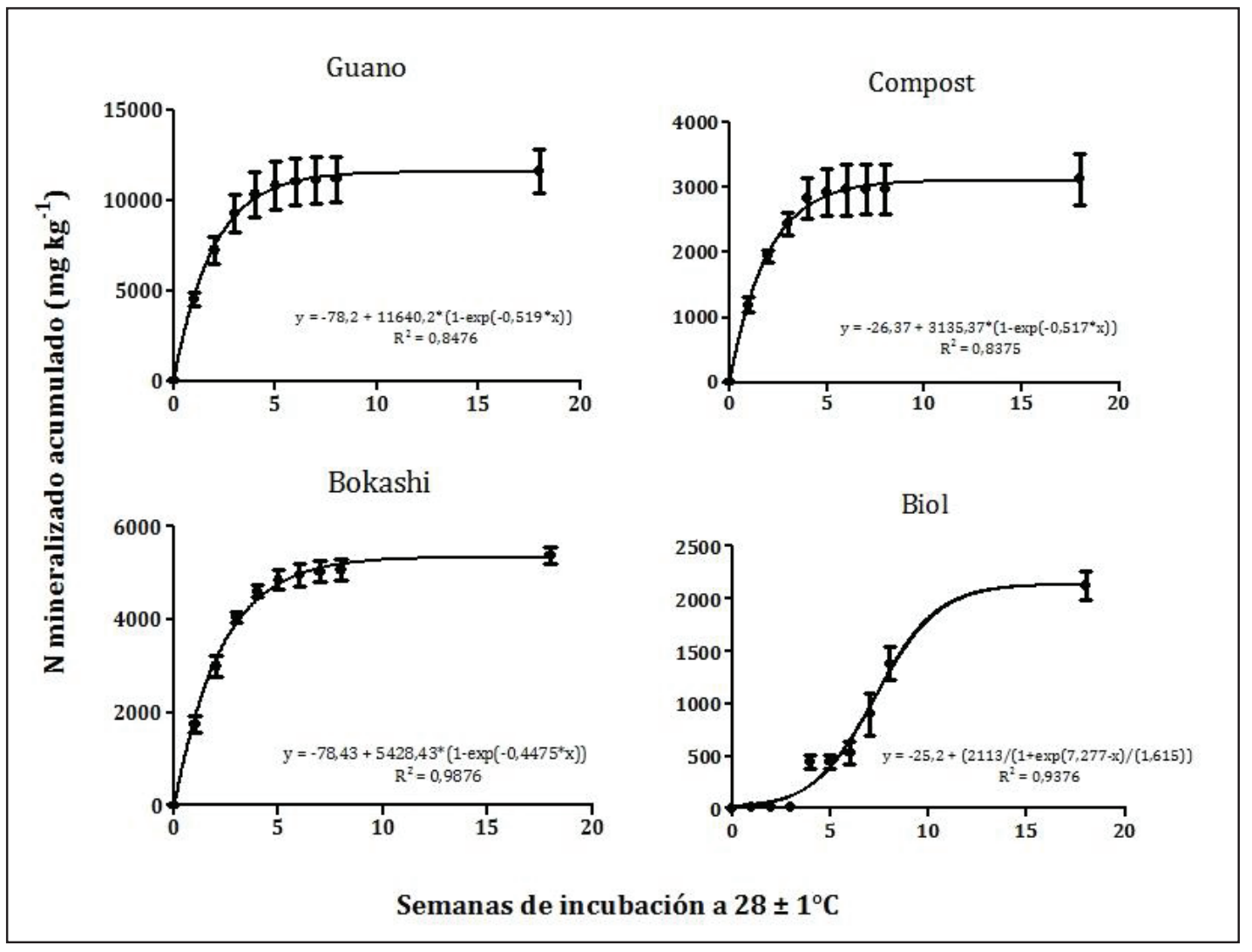

Figura 3. Mineralización acumulada promedio de nitrógeno en guano, compost, bokashi y biol. Barras verticales muestran error estándar de la media.

Figure 3. Average accumulated nitrogen mineralization in poultry, compost, bokashi and biol. Vertical bars show standard error of the mean.

riales orgánicos ricos en Ca en la xilosa y arabinosa de los rastrojos del trigo (Le et al., 2014) que se utilizaron en el estudio para la preparación de compost y bokashi.

Los valores de $\mathrm{Cu}$ y $\mathrm{Zn}$ en los abonos evaluados fueron menores a $100 \mathrm{mg} \mathrm{kg}^{-1}$, que es el valor crítico propuesto por Alloway (2013) para materiales orgánicos. Estos contenidos cumplen, también, con los requisitos para la definición de calidad de los abonos orgánicos y adicionalmente presentaron una baja concentración en $\mathrm{Na}$ (promedio de $0,1 \%$ ), usual de acuerdo a su origen de guano de gallina.

El contenido de MO de los abonos orgánicos disminuyó cuando se incrementó el tiempo de preparación. Ello es atribuido al incremento de la acción microbial lo que produce respiración y una disminución del contenido de C. Durante este proceso los abonos, que alcanzan mayores temperaturas, como compost y bokashi presentaron menores contenidos de MO (Zbytniewski y Buszewski, 2005; Ros et al., 2006). Mayores contenidos de MO se presentaron en guano y biol, que estuvieron a temperatura ambiente durante todo el tiempo previo a su incubación, dando como resultado una menor mineralización de los productos originarios, dado por una menor actividad microbiana (Tiquia et al., 1998).

\section{Acidez, conductividad eléctrica, relación $\mathrm{C} / \mathrm{N}$ y contenido de nitrógeno mineral inicial $(\mathrm{Nmi})$}

Los valores de $\mathrm{pH}$ cercanos a la neutralidad de los tres abonos orgánicos sólidos estudiados han sido atribuidos a una estabilización de la MO original (Sesay et al., 1997). Sin embargo, más recientemente se ha indicado que la acidez no implicaría estabilidad de la MO, ya que los valores de acidez son dependientes de los materiales iniciales ocupados en la elaboración del abono (Said-Pullicino et al., 2007). Para el compost, pH más alcalino inicial puede deberse a una posible liberación de $\mathrm{N}$ en forma amoniacal, en el periodo de altas temperaturas, lo cual afectaría el equilibrio ácido-base influyendo en la conservación del $\mathrm{N}$ en el medio (Tiquia y Tam, 2002) antes de transformarse a nitrato. Esta situación, podría explicar también los menores contenidos de $\mathrm{N}$ presentes en compost y bokashi respecto al 


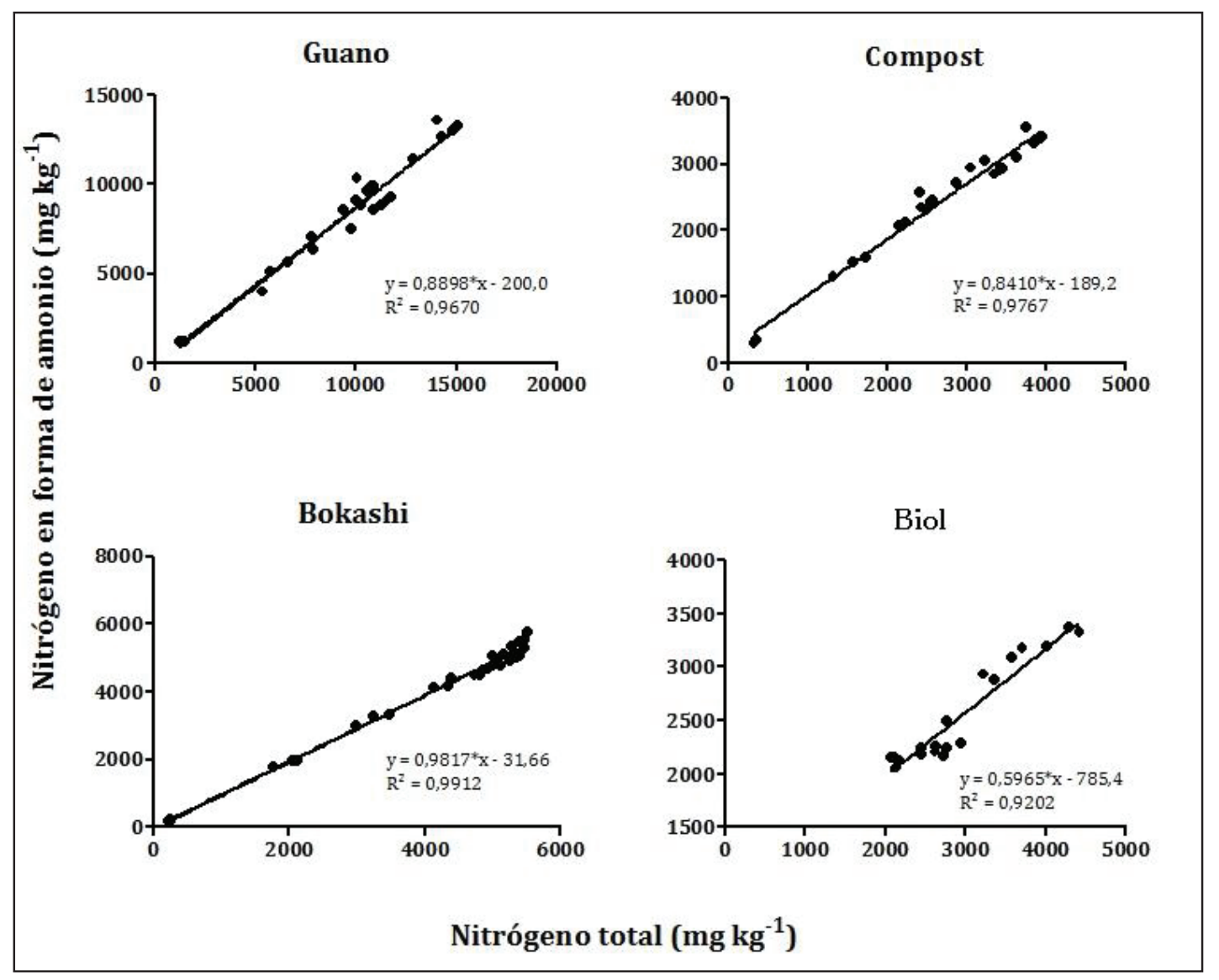

Figura 4. Relación entre el nitrógeno mineralizado total acumulado respecto al mineralizado en forma de amonio.

Figure 4. Relationship between the accumulated total mineralized nitrogen with respect to the mineralized in the form of ammonium.

guano de gallina. En el caso de biol, su pH más ácido, podría deberse a un mayor contenido de nitratos en el $\mathrm{Nmi}$, lo que es debido a la nitrificación del $\mathrm{N}$ amoniacal, como se muestra en su mayor proporción de nitratos que los otros abonos orgánicos. Además, sería producto de una mayor actividad biológica en el fermento y una mayor acumulación de ácidos grasos volátiles, en el fermentador. Valores de $\mathrm{pH}$ ácidos similares a los reportados aquí, fueron determinados por Guanopatín (2012) al estudiar distintos tipos de biol. También, los componentes originales adicionados en la preparación de biol podrían acelerar su cambio de pH. Así la presencia de la leche (suero) sería causal de la baja de $\mathrm{pH}$ (Ito, 2006).

La CE de los abonos producidos fue similar a la determinada por Cáceres et al. (2006) en otros abonos orgánicos. Bokashi presentó una CE similar al guano y el leve incremento de la CE para compost se explica por pérdida de masa, durante la mineralización de la MO, aumentando la concentración de sales. La relación lineal entre $\mathrm{CE}$ y $\mathrm{pH}$ fue similar a la determinada para otros abonos orgánicos (Ito, 2006). En este estudio, biol fue el único abono que presentó una alta CE, cinco veces mayor que los otros abonos orgánicos, lo que estuvo asociado a una mayor cantidad de partículas en suspensión, presente en este abono producido a través de fermentación y diferente a los otros abonos que son producto de una oxidación.

Las relaciones $\mathrm{C} / \mathrm{N}$ de los 4 abonos mostraron que todos tienen suficiente $\mathrm{N}$ para satisfacer las necesidades de los microorganismos degradantes (HernándezRodríguez et al., 2013), lo que es probablemente debido a su contenido de guano de gallina como material base (Zmora-Nahum et al., 2005).

El $\mathrm{N}$ mineralizado en el tiempo cero parece relacionarse positivamente con el contenido de MO de los abonos orgánicos evaluados y con el porcentaje de $\mathrm{N}$ orgánico contenido. También hay que considerar que hubo efecto de dilución del contenido de $\mathrm{N}$ y MO al preparar los abonos orgánicos, por lo que la calidad base dada por el guano de gallina se debe comparar considerando contenidos similares. De esta manera, abonos con mayores contenidos MO y N (biol y guano) presentarían mayores fracciones de $\mathrm{N}$ rápidamente mineralizable 
por los microorganismos (Hernández-Rodríguez et al., 2013), siendo biol y guano, la mejor opción para agregar mayor cantidad de $\mathrm{N}$ mineral en forma inmediata. Las cantidades menores presentadas en el compost y el bokashi, son concordantes con una fase de maduración de los abonos, caracterizada por un lento proceso de mineralización limitado por el suministro de MO (Tognetti et al., 2007).

\section{Mineralización potencial del nitrógeno}

Las curvas ajustadas de los abonos sólidos para la mineralización acumuladas son similares a las determinadas por Rodríguez y Matus (1989) en suelos, por Kameko (2003) en abonos orgánicos y Artacho et al. (2001) en lodos y guano. La fase de adaptación inicial (lag) previa antes de establecerse la fase exponencial presente en el biol, se debe a la necesidad de un crecimiento microbial, antes de establecer una fase de enzima constante en la degradación de la MO (Bohn, et al., 1993; Alexander, 1980). En los abonos orgánicos sólidos, la mineralización fue mayor al inicio de la incubación, debido al mayor contenido de compuestos metabólico celulares, disminuyendo su velocidad hacia las últimas semanas, donde se mineralizan compuestos estructurales más resistentes como hemicelulosa, celulosa y lignina (Figueroa-Barrera et al., 2012). La tasa de mineralización de los abonos orgánicos evaluados presentó tres fases en el tiempo: i) las dos primeras semanas con una mayor tasa de mineralización, b) entre la segunda y quinta semana, la tasa de mineralización puede describirse como intermedia, y c) desde la quinta semana en adelante, donde la tasa de mineralización tiende a disminuir hasta estabilizarse. La segunda y tercera fases pueden ser la condicionada por los sustratos adicionales al guano agregado y su contenido relativo de lignina (Rodríguez y Matus, 1989).

En este estudio, el proceso de amonificación no fue limitado por $\mathrm{pH}$, humedad y temperatura. Esto se vería reflejado en la naturaleza residual de los componentes orgánicos de los abonos (Frioni, 1999). El comportamiento diferente del biol, tanto en su baja cantidad de $\mathrm{N}$ mineralizado y su demora inicial en la mineralización pudo deberse al cambio desde condiciones anaeróbicas a aeróbicas. Ello produciría una inmovilización del $\mathrm{N}$ durante la incubación, al producirse una nueva biomasa microbial. Es por ello, que en futuras investigaciones, se debería cuantificar la población y los tipos de microorganismos presente en los abonos orgánicos. Además, es importante prolongar el periodo de mineralización, acotando la frecuencia de medición, lo que permitiría conocer el tiempo final de la primera fase y ayudaría a tener una mayor precisión para hacer coincidir la máxima tasa de mineralización con las etapas del cultivo donde se demanden mayores cantidades de $\mathrm{N}$.

\section{Relación del Npm total respecto al contenido de amonio}

Los altos valores de $\mathrm{NH}_{4}$ medidos en los cuatro abonos orgánicos se encuentran dentro de los rangos descritos por Kelleher (2002), quien los atribuye a las altas concentraciones $\mathrm{N}$ orgánico que se encuentran en el guano de gallina en forma de proteínas y aminoácidos. Los abonos orgánicos que presentan una mayor relación $\mathrm{N}-\mathrm{NH}_{4}$ y $\mathrm{N}$ total podrían encontrarse degradando la MO en forma activa (Tiquia et al., 1998). Si esto fuera así, se podría ordenar los abonos orgánicos según su actividad microbial, encontrándose en primer lugar bokashi, el guano, compost y biol. Sin embargo, para conocer esto se requeriría medir evolución de $\mathrm{CO}_{2}$, cantidad de biomasa microbial y/o actividad enzimática (Pinochet et al., 2014). En futuras investigaciones se recomienda evaluar la relación amonio nitrato como un indicador de madurez de los abonos orgánicos y cuantificar las pérdidas de $\mathrm{N}$ por volatilización en forma de $\mathrm{NH}_{3}$, debido a que estas tienden a ser frecuentes en compost con pH superiores a 7 (DeLaune et al., 2004).

\section{CONCLUSIONES}

La composición base de los fertilizantes orgánicos en macronutrientes, fue similar entre guano y biol; por su parte, compost y bokashi presentaron menores concentraciones, atribuibles al porcentaje de participación del guano en su elaboración. Los niveles de micronutrientes, dependieron del tipo de material vegetal que acompaña el material base en la elaboración, siendo mayores en compost y bokashi. El contenido de MO fue variable entre los abonos orgánicos, debido al tiempo y condiciones de elaboración.

La cantidad total de $\mathrm{N}$ potencialmente mineralizable fue mayor en el guano que los demás abonos orgánicos, los cuales presentan un mayor contenido de compuestos estructurales resistentes en su elaboración. Los cuatro abonos orgánicos presentaron relaciones lineales entre el Npm total respecto al Npm en forma de amonio y de los cuatro abonos, el biol fue quien presentó una mayor mineralización en forma de nitrato. Los resultados obtenidos en el trabajo son atribuibles a los procesos de elaboración descritas. Abonos orgánicos con los mismos nombres, pero con distintos materiales y procesos de elaboración podrían mostrar resultados diferentes.

Para estudios posteriores es importante evaluar la existencia de una correlación entre contenido de nutrientes en el suelo y algunos elementos minerales estudiados, especialmente para bokashi y compost, los cuales pueden variar en el tipo de suelo y en las cantidades usadas en su preparación, lo que afectar su concentración final de minerales. 


\section{REFERENCIAS}

Alexander, M., 1980. Introducción a la Microbiología de Suelo. AGT Editor. México.

Alloway, B.J., 2013. Heavy metals in soils. Trace metals and metalloids in soils and their bioavailability. $3^{\text {th }}$ Edition. Environmental Pollution 22. Springer, London.

Altieri, M.A., Nicholls, C.I., 2012. Agroecología: única esperanza para la soberanía alimentaria y la resiliencia socioecológica. Agroecología 7(2), 65-83.

Artacho, P., Pinochet, D., Azúa P., 2001. Potencialidad como abono orgánico de los desechos sólidos subproductos del cultivo de especies salmonídeas. Agro Sur 29, 78-82.

Barrera, A.F., Herrera, J.G.Á., Forero, A.F., Salamanca, C., Pinzón, L.P., 2012. Determinación del Nitrógeno potencialmente mineralizable y la tasa de mineralización de nitrógeno en materiales orgánicos. Temas Agrarios 17(1), 32-43.

Barbaro, L.A., Karlanian, M.A., Rizzo, P.F., Riera, N.I., Della Torre, V., Beltrán, M., Crespo, D.E., 2013. Compost de guano de gallina en la composición de sustratos para la producción de plantines florales. Agriscientia 30(1), 25-35.

Bohn, H., McNeal, B. O’Connor, G., Sánchez Orozco, M., 1993. Química del Suelo. Editorial Limusa. México.

Cáceres, R., Flotats, X., Marfà, O., 2006. Changes in the chemical and physicochemical properties of the solid fraction of cattle slurry during composting using different aeration strategies. Waste Management 26(10), 1081-1091.

Castillo, A., Quarín, S., Iglesias, M., 2000. Vermicompost chemical and physical characterization from raw and mixed organic wastes. Agricultura Técnica 60, 74-79.

Clivot, H., Mary, B., Valé, M., Cohan, J.P., Champolivier, L., Piraux, F., Laurent, F., Justes, E., 2017. Quantifying in situ and modeling net nitrogen mineralization from soil organic matter in arable cropping systems. Soil Biology and Biochemistry 111, 44-59.

DeLaune, P.B., Moore, P.A., Daniel, T.C., Lemunyon, J.L., 2004. Effect of chemical and microbial amendments on ammonia volatilization from composting poultry litter. Journal of Environmental Quality 33(2), 728-734.

Díaz, M.J., Madejón, E., Cabrera, F., Jiménez, L., De Bertoldi, M., 2004. Using a second-order polynomial model to determine the optimum vinasse/grape marc ratio for in-vessel composting. Compost Science and Utilization 12(3), 273-279.

Figueroa-Barrera, A., Álvarez-Herrera, J., Forero, A., Salamanca, C., Pinzón, L., 2012. Determinación del nitrógeno potencialmente mineralizable y la tasa de mineralización de nitrógeno en materiales orgánicos. Temas Agrarios 17(1), 32-43.

Frioni, L., 1999. Procesos microbianos. Tomo I y II. Editorial de la fundación UNRC. Universidad Nacional de Río Cuarto, Argentina.

Guanopatín, M., 2012. Aplicación de biol en el cultivo establecido de alfalfa (Medicago sativa). Tesis Lic. Agr. Universidad Técnica de Ambato Ecuador, Facultad de Ciencias Agrarias. 93 p.

Havlin, J.L., Kissel, D.E., Maddus, L.D., Claassen, M.M., Long J.H., 1990. Crop rotation and tillage effects on soil organic carbon and nitrogen. Soil Science Society of American Journal 54, 448-452.
Hernández-Rodríguez, O.A., Hernández-Tecorral, A., Rivera-Figueroa, C., Arras-Vota, A.M., Ojeda-Barrios, D., 2013. Calidad nutrimental de cuatro abonos orgánicos producidos a partir de residuos vegetales y pecuarios. Terra Latinoamericana 31(1), 35-46.

Infante, A., 2011. Manual de biopreparados para la agricultura ecológica. Programa territorial orgánico (PTO), SURFRUT, Fundación para la Innovación Agraria (FIA). Trama impresores S.A, Santiago, Chile.

Ito, S., 2006. Caracterización y evaluación de los factores que determinan la calidad nutricional e inocuidad en la producción de fertilizantes orgánicos fermentados. Tesis Magister Agricultura Ecológica. Centro Agronómico Tropical de Investigación y Enseñanza. 119 p.

Kameko, C., 2003. Determinación del potencial de mineralización del nitrógeno de bocachi, compost y lombricompost producidos en la Universidad Earth. Tesis Lic. Agr. Guácimo. Universidad Earth Costa Rica, Facultad de Agronomía. $50 \mathrm{p}$.

Kelleher, B.P., Leahy, J.J., Henihan, A.M., O’Dwyer, T.F., Sutton, D., Leahy, M.J., 2002. Advances in poultry litter disposal technology- A review. Bioresource Technology 83(1), 27-36.

Jones, J.W., Antle, J.M., Basso, B., Boote, K.J., Conant, R.T., Foster, I., Godfray, H.C.J., Herrero, M., Howitt, R.E., Janssen, S., Keating, B.A., Muñoz-Carpena, R., Porter, C.H., Rosenzweig, C., Wheeler, T.R., 2017. Toward a new generation of agricultural system data, models, and knowledge products: State of agricultural systems science. Agricultural Systems 155, 269-288.

Le, D.M., Sørensen, H.R., Knudsen, N.O., Schjoerring, J.K., Meyer, A.S., 2014. Biorefining of wheat straw: accounting for the distribution of mineral elements in pretreated biomass by an extended pretreatment-severity equation. Biotechnology for Biofuels 7(1), 141.

Leblanc, H.A., Cerrato, M.E., Miranda, A., Valle, G., 2007. Determinación de la calidad de abonos orgánicos a través de bioensayos. Tierra Tropical 3(1), 97-107.

Pérez, A., Céspedes, C., Núñez, P., 2008. Caracterización físicaquímica y biológica de enmiendas orgánicas aplicadas en la producción de cultivos en República Dominicana. Revista de la Ciencia del Suelo y Nutrición Vegetal 8(3), 10-29.

Pinochet, D., Mendoza, J., Galvis, A., 2000. Potencial de mineralización de nitrógeno de un Hapludand con distintos manejos agrícolas. Ciencia e Investigación Agraria 27(2), 97-106.

Pinochet, D., Valenzuela, E., Solís, J., Clunes, J., 2014. Mineralización y/o solubilización del fósforo contenido en lodos provenientes de residuos salmonícolas marinos bioaumentados con cepas de Aspergillus niger y Trichoederma pseudokoningi. Boletín Micológico 29(2), 46-55.

Ramos, D., Terry, E., 2014. Generalidades de los abonos orgánicos: importancia del bocashi como alternativa nutricional para suelos y plantas. Cultivos Tropicales 35(4), 52-59.

Restrepo, J., Hensel, J., 2009. Manual práctico de agricultura orgánica y panes de piedra. $1^{\text {a }}$ Edición, Feriva S.A. Cali, Colombia.

Restrepo, J., 2007. Manual práctico: El A, B, C de la agricultura orgánica y harina de rocas. $1^{\circ}$ edición. http://www.utn. org.mx/docs_pdf/capacitacion_tecnica_2009/manua- 
les/agricultura_ecologica/manual_practico_abc_agricultura_organica.pdf (acceso, 24.03. 2013).

Rodríguez, J., Matus, F., 1989. Modelo simple para estimar el suministro de nitrógeno en el suelo. Revista Ciencia e Investigación Agraria 16, 33-46.

Ros, M., García, C., Hernández, T., 2006. A full-scale study of treatment of pig slurry by composting: kinetic changes in chemical and microbial properties. Waste Management 26, 1108-1118.

Sadzawka, A., Carrasco, M., Grez, R., Mora, M., 2005. Métodos de análisis de compost. Revisión 2005. Métodos de análisis de compost. Instituto de Investigaciones Agropecuarias, Centro Regional de Investigación La Platina, Serie Actas INIA N 30, Santiago, Chile.

Sadzawka, A., Carrasco, M., Grez, R., Mora, M., Flores, H., Neaman, A., 2006. Métodos de análisis recomendados para los suelos de Chile. Instituto de Investigaciones Agropecuarias. Serie Actas INIA N ${ }^{\circ}$ 34, Santiago, Chile.

Servicio Agrícola y Ganadero (SAG), 2013. Agricultura orgánica nacional, bases técnicas y situación actual. 1a Edición. http://www.sag.cl/sites/default /files/agricultura_ org._nacional_bases_tecnicas_y_situacion_actual_2013. pdf (acceso, 20.06. 2015).

Said-Pullicino, D., Erriquens, F., Gigliotti, G., 2007. Changes in the chemical characteristics of water-extractable organic matter during composting and their influence on compost stability and maturity. Bioresource Technology 98, 1822-1831.

Sesay, A.A., Lasaridi, K., Stentiford, E., Budd, T., 1997. Controlled composting of paper pulp sludge using the aerated static pile method. Compost Science and Utilization 5(1), 82-96.
Standford, G., Smith, S.J., 1972. Nitrogen mineralization potentials of soil. Soil Science Society of America Proceedings 36, 465-472.

Suquilandia, M., 1996. Agricultura orgánica, alternativa tecnológica del futuro. Editorial Abya Yala. Quito, Ecuador.

Tiquia, S.M., Tam, S., 2002. Characterization and composting of poultry litter in forced-aeration piles. Process Biochemistry 37, 869-880.

Tiquia, S.M., Tam, N.F.Y., Hodgkiss, I.J., 1998. Changes in chemical properties during composting of spent pig litter at different moisture contents. Agriculture, Ecosystems and Environment 67(1), 79-89.

Tognetti, C., Mazzarino, M.J., Laos, F., 2007. Cocomposting biosolids and municipal organic waste: effects of process management on stabilization and quality. Biology and Fertility of Soils 43(4), 387-397.

Varnero, M., Gonzáles, P., Silva, G., 2002. Avances en restauración ambiental con énfasis en recuperación ecológica. Proyecto FONDEF d9811036. Universidad de Chile, Facultad de Ciencias Forestales, Departamento Manejo de Recursos Forestales. Publicaciones Misceláneas Forestales № 4. Santiago, Chile.

Yagodín, B., Smirnov, P., Peterburgs, K., 1982-1986. Agroquímica, tomos I y II. Editorial Mir, Moscú.

Zbytniewski, R., Buszewski, B., 2005. Characterization of natural organic matter (nom) derived from sewage sludge compost. Part 1: Chemical and Spectroscopic Properties. Bioresource Technology 96, 471-478.

Zmora-Nahum, S., Markovitch, O., Tarchitzky, J., Chen, Y., 2005. Dissolved organic carbon (DOC) as a parameter of compost maturity. Soil Biology and Biochemistry 37(11), 2109-2116. 